\title{
Association of Body Mass Index with All-Cause and Cardiovascular Disease Mortality in the Elderly
}

\section{Chen-Yi Wu ${ }^{1,2}$, Yi-Chang Chou ${ }^{1,3}$, Nicole Huang ${ }^{3,4}$, Yiing-Jenq Chou ${ }^{1}$, Hsiao-Yun Hu ${ }^{1,3 *}$, Chung-Pin Li ${ }^{5,6 *}$}

1 Institute of Public Health, National Yang Ming University, Taipei, Taiwan, 2 Department of Dermatology, Taipei City Hospital, Heping Fuyou Branch, Taipei, Taiwan, 3 Department of Education and Research, Taipei City Hospital, Taipei, Taiwan, 4 Institute of Hospital and Health Care Administration, National Yang-Ming University, Taipei, Taiwan, 5 Division of Gastroenterology, Department of Medicine, Taipei Veterans General Hospital, Taipei, Taiwan, 6 National Yang-Ming University School of Medicine, Taipei, Taiwan

\begin{abstract}
Objectives: To evaluate the associations of body mass index (BMI) with all-cause, cardiovascular disease (CVD), and expanded CVD mortality in the elderly.
\end{abstract}

Design: Observational cohort study.

Setting: Annual physical examination program for the elderly from 2006 to 2010.

Participants: We included 77,541 Taipei residents aged $\geq 65$ years (39,365 men and 38,176 women).

Measurements: $\mathrm{BMI}$ was categorized as underweight $(\mathrm{BMI}<18.5)$, normal weight $(18.5 \leq \mathrm{BMI}<25)$, overweight $(25 \leq \mathrm{BMI}<$ $30)$, grade 1 obesity $(30 \leq \mathrm{BMl}<35)$, or grade $2-3$ obesity $(\mathrm{BMI} \geq 35)$. Mortality was ascertained by national death files.

Results: Underweight (hazard ratios [HRs] of all-cause, CVD, and expanded CVD mortality: 1.92, 1.74, and 1.77, respectively), grade $2-3$ obesity (HRs: 1.59, 2.36, and 2.22, respectively), older age, male sex, smoking, and high fasting blood sugar were significant predictors of mortality. Meanwhile, being married/cohabitating, higher education, alcohol consumption, more regular exercise, and high total cholesterol were inversely associated with mortality. Multivariate stratified subgroup analyses verified smokers (HRs of all-cause, CVD, and expanded CVD mortality: 3.25, 10.71, and 7.86, respectively, for grade 2-3 obesity), the high triglyceride group (HRs: 5.82, 10.99, and 14.22, respectively for underweight), and patients with 3-4 factors related to metabolic syndrome (HRs: 4.86, 12.72, and 11.42, respectively, for underweight) were associated with mortality.

Conclusion: The associations of BMI with all-cause, CVD, expanded CVD mortality in the elderly are represented by Ushaped curves, suggesting unilateral promotions or interventions in weight reduction in the elderly may be inappropriate. Heterogeneous effects of grades 1 and 2-3 obesity on mortality were observed and should be treated as different levels of obesity.

Citation: Wu C-Y, Chou Y-C, Huang N, Chou Y-J, Hu H-Y, et al. (2014) Association of Body Mass Index with All-Cause and Cardiovascular Disease Mortality in the Elderly. PLoS ONE 9(7): e102589. doi:10.1371/journal.pone.0102589

Editor: Salomon Amar, Boston University, United States of America

Received December 16, 2013; Accepted June 20, 2014; Published July 11, 2014

Copyright: (c) 2014 Wu et al. This is an open-access article distributed under the terms of the Creative Commons Attribution License, which permits unrestricted use, distribution, and reproduction in any medium, provided the original author and source are credited.

Funding: This study was supported by the Taiwan Ministry of Education through its "Aim for the Top University Plan," by the Department of Health of the Taipei City Government (Grant 10301-62-001), and by Taiwan's National Science Council (Grant 98-2314-B-010-015-MY2). This study is based on data from the Taipei City Public Health Database provided by the Department of Health of the Taipei City Government and managed by the Databank for Public Health Analysis. The funders had no role in study design, data collection and analysis, decision to publish, or preparation of the manuscript.

Competing Interests: The authors have declared that no competing interests exist.

*Email: hyhu@ym.edu.tw (HYH); cpli@vghtpe.gov.tw (CPL)

\section{Introduction}

Overweight/obesity has become a serious public health problem accompanying changes in dietary habits and physical activity and is increasing to epidemic proportions in most industrialized counties [1,2]. Many in-depth studies have investigated the impacts of body weight on mortality. However, the elderly are frequently excluded from such studies, particularly those presenting with compromising comorbidities. Cardiovascular disease (GVD) represents the main cause of mortality in the elderly population; obesity is a significant risk factor for CVD and other CVD-related diseases such as hypertension, type II diabetes mellitus, and dyslipidemia [3],[4]. Nevertheless, clarifying how overweight or obesity influences the survival of the elderly, including all-cause and CVD mortality, may be critical.

Numerous reports in the previous decade document an obesity paradox: overweight or obese individuals are reported to have a mortality risk lower than that of normal weight individuals [5-10]. This contradicts the well-accepted empirically based idea that obesity confers increased mortality. This controversy is frequently debated, and whether such findings can be applied to the elderly, which is the fastest growing population segment in many countries, remains inconclusive. Body mass index (BMI) is a reasonably good measure of general adiposity in the elderly [2]. The effects of 
increasing BMI on mortality are less pronounced in the elderly than young or middle-aged adults [11]. However, another study reports the obesity-mortality relationship strengthens with age after controlling for confounders [12]. In 2006, Janssen et al. performed a meta-analysis examining the association between elevated BMI and all-cause mortality in the elderly; they report overweight is not associated with a significantly increased risk of mortality, while moderate obesity is associated with a modest increase in risk [13]. In contrast, in 2013, Flegal et al. performed another meta-analysis evaluating the associations of overweight and obesity with all-cause mortality in the elderly; they report overweight is associated with significantly lower mortality and obesity is not associated with higher mortality [14]. Meanwhile, Lin et al. report higher BMI and waist circumference are associated with lower mortality in Chinese long-term care facility residents [15]. These contradictory findings may be attributable to the different follow-up periods and covariates analyzed [16]. Thus, the optimal BMI and effects of being underweight or overweight on the risk of mortality of the elderly remain controversial. Therefore, further evidence is needed to clarify this issue.

This study evaluated the associations of BMI with all-cause mortality, CVD mortality, and expanded CVD in a large cohort of elderly Taiwanese people, who were medically screened in a standardized process and followed-up for 5 years, yielding 254,211 person-years of observation. Expanded CVD was coined as a new category to capture additional deaths traditionally not classified as CVDs, such as diabetes and kidney diseases.

\section{Methods}

\section{Study population}

The main data were from the Taipei Geriatric Health Examination Database. The study cohort comprised 77,541 participants aged 65 years or older, including 39,365 men and 38,176 women, in a standard annular physical examination program for elderly people run by the Taipei City Government from 2006-2010. Identical screening procedures and protocol were used in all qualified hospitals that contracted with the Department of Health, Taipei City Government [17]. The results were centrally managed and stored. Subjects participated in the physical examination program voluntarily and were encouraged to visit on an annual basis. However, only the results from the initial visit were analyzed. Demographic and lifestyle information (e.g., marital status, education level, smoking history, alcohol consumption, and exercising habit) were collected through a selfadministered questionnaire. During the medical check-up, blood pressure was measured. Overnight fasting blood was collected for the measurement of serum blood sugar, triglyceride (TG), total cholesterol (TC), glutamic oxaloacetic transaminase (GOT), glutamic pyruvic transaminase (GPT), albumin, blood urine nitrogen (BUN), creatinine, and hemoglobin. Data related to individual identification were removed before all data were released to the researchers. The acquisition and processing of the data were approved by the Institutional Review Board of Taipei City Hospital (approval number: TCHIRB-1020417-E).

\section{Definition of BMI}

Height and weight were measured during examinations using standardized procedures. BMI was calculated as weight in kilograms divided by height in meters squared. This study employed the BMI-based categories defined by the World Health Organization $(\mathrm{WHO})$ of underweight $(\mathrm{BMI}<18.5)$, normal weight $(18.5 \leq \mathrm{BMI}<25$, reference category), overweight $(25 \leq \mathrm{BMI}<30)$, grade 1 obesity $(30 \leq \mathrm{BMI}<35)$, and grade $2-3$ obesity $(\mathrm{BMI} \geq 35)$.

\section{Controlled variables}

Baseline data were collected, including age (i.e., 65-69, 70-74, 75-79, 80-84, or $\geq 85$ years), sex, marital status (i.e., single, married/cohabiting), education level (i.e., none, 1-6 years, 7-12 years, or $>12$ years), smoking (i.e., frequently/occasionally or no), alcohol consumption (i.e., frequently/occasionally or no), and regular exercise (i.e., none, 1-2 times/week, or 3-5 times/week), high fasting blood sugar (FBS) (i.e., no or $\geq 126 \mathrm{mg} / \mathrm{dL}$ ), high systolic blood pressure (SBP) (i.e., no or $\geq 140 \mathrm{mmHg}$ ), high TG (i.e., no or $\geq 200 \mathrm{mg} / \mathrm{dL}$ ), and high TC (i.e., no or $\geq 200 \mathrm{mg} / \mathrm{dL}$ ).

\section{Outcome variables}

The vital status of the 77,541 study subjects as of December 31 , 2010 was ascertained by matching cohort IDs with computerized national death files. Information on the causes of death was coded according to the International Classification of Diseases, Ninth Revision (ICD-9) 2006 or ICD-10 2009 and 2010, including all causes (ICD-9: 001-998; ICD-10: A00-Z99), CVDs (ICD-9: 390459; ICD-10: I00-I99), and expanded CVD (CVD plus diabetes, ICD-9: 250; ICD-10: E10-E14, plus kidney diseases, ICD-9: 580589; ICD-10: N00-N29).

\section{Statistical analyses}

The relative risks of all-cause, GVD, and expanded CVD mortality were calculated using Cox proportional hazards models. The proportional hazard assumption was examined by plotting the log-log plots of each category of BMI, and the plot showed that the line for each category was straight and parallel. We also tested whether the log hazard ratio function is constant over time. For 19 of the 21 variables, we accepted the null hypothesis, and for 2 variables, we rejected the null hypothesis, i.e., exercise for 3-5 times/week $(P<0.001)$ and BMI of $25-29.9 \mathrm{~kg} / \mathrm{m}^{2}(P=0.03)$. The log-log plots indicated that the data might be suitable for the Weibull distribution parameters estimation. A sensitivity test of the Weibull model was performed. The times of entry and exit were the date of examination and the end of follow-up (December 31, 2010 ) or death if earlier, respectively. The reference group comprised subjects with $18.5 \leq \mathrm{BMI}<25$, and the hazard ratio (HR) for each BMI category was calculated. Two models were used to avoid over-adjusting for factors in the causal relationship between obesity and mortality. The first model was adjusted for age, sex, marital status, education level, smoking, alcohol consumption, and physical activity; models adjusted for these factors are considered to be adequately adjusted [14]. Additional variables closely related to metabolic syndrome, including high FBS, high SBP, high TG, and high TC, were adjusted for in the second model, which was considered an over-adjusted model. Furthermore, the HRs were calculated with grades 1 and 2-3 obesity combined as a single obesity group. Another sensitivity test was performed to calculate the HR with the reference group set as subjects with $23 \leq \mathrm{BMI}<25$.

In the model considered adequately adjusted, HRs were calculated for the following specific subgroups: non-smokers, smokers, men, women, young-old (65-74 years), old-old ( $\geq 75$ years), normal FBS, high FBS ( $\geq 126 \mathrm{mg} / \mathrm{dL}$ ), normal SBP, high SBP $(\geq 140 \mathrm{mmHg})$, normal TG, high TG $(\geq 200 \mathrm{mg} / \mathrm{dL})$, normal TC, and high TC ( $\geq 200 \mathrm{mg} / \mathrm{dL})$ as well as and $0,1,2$, or 3-4 risk factors for metabolic syndrome (i.e., high FBS, high SBP, high TG, and high TG).

All analyses were performed using SAS 9.3 (SAS Institute Inc., Cary, NG, USA) and the STATA 10.0 (STATA Corp, College Station, TX, USA). The level of significance was set at $P<0.05$. 


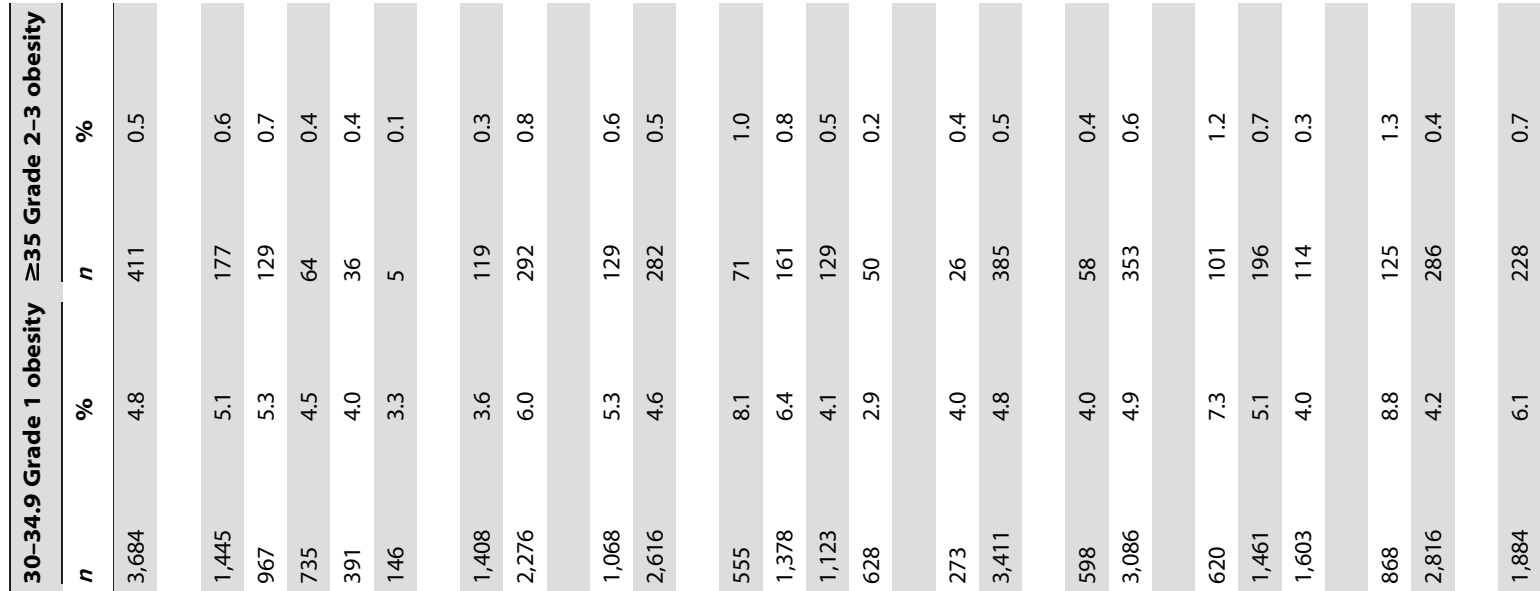

帝

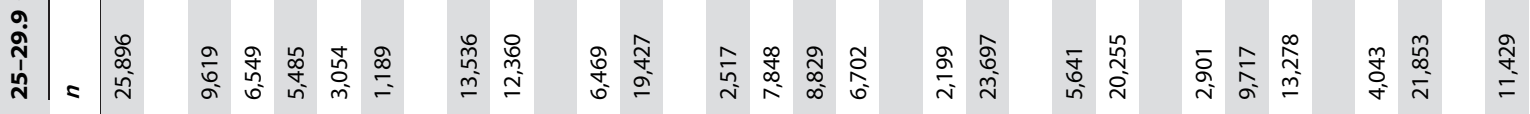

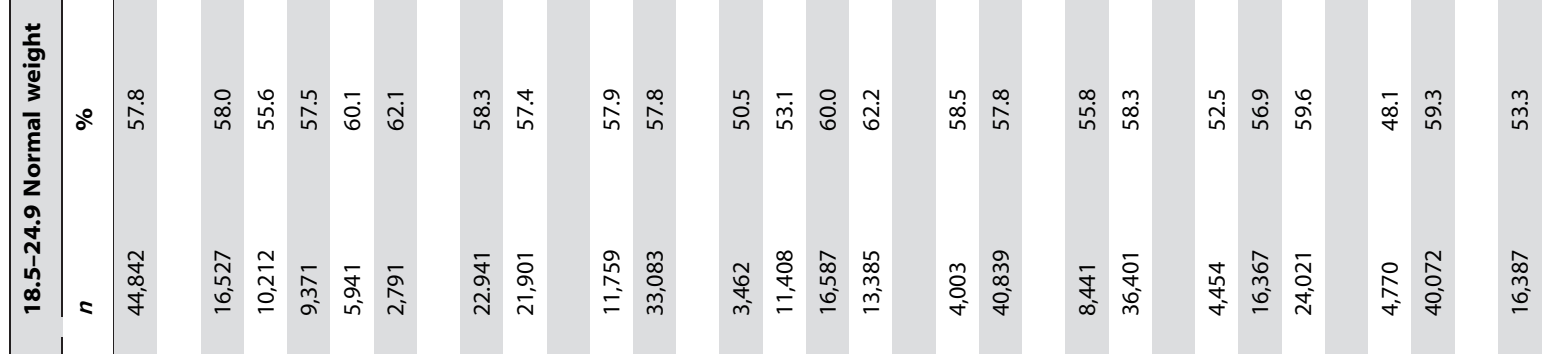

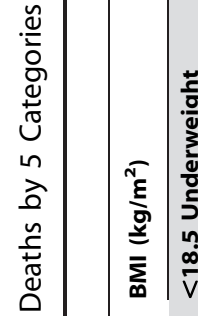

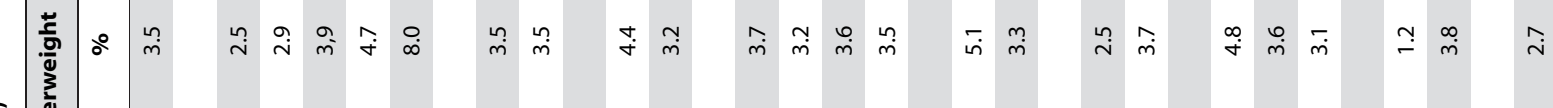

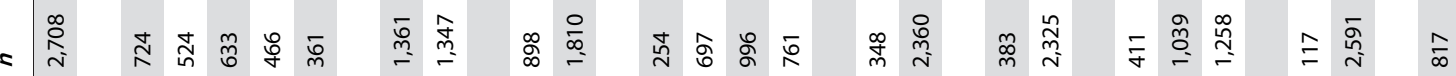

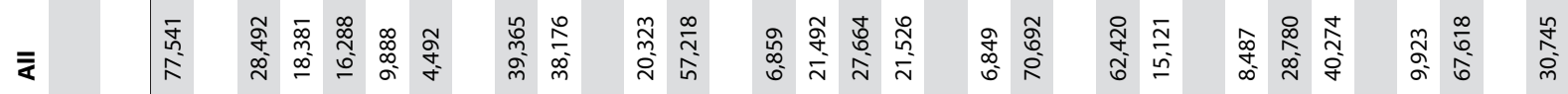




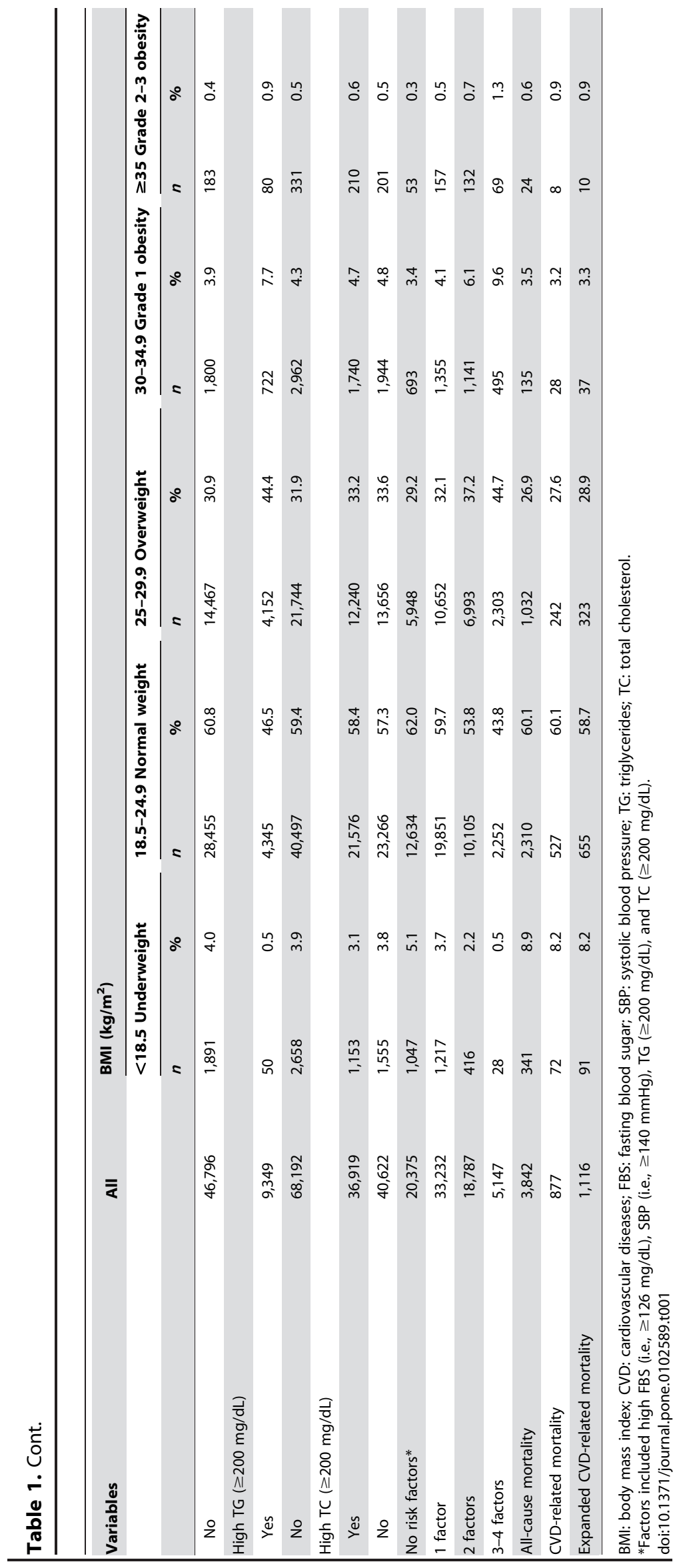


Table 2. Clinical Characteristics of Participants According to the BMI Category.

\begin{tabular}{|c|c|c|c|c|c|c|}
\hline \multirow{4}{*}{ Variables } & \multirow{4}{*}{$\begin{array}{l}\text { All } \\
\text { Mean } \pm \text { SD }\end{array}$} & \multicolumn{5}{|l|}{ BMI $\left(\mathbf{k g} / \mathbf{m}^{2}\right)$} \\
\hline & & \multirow{2}{*}{$\frac{<18.5}{\text { Underweight }}$} & \multirow{2}{*}{$\frac{18.5-24.9}{\text { Normal weight }}$} & \multirow{2}{*}{$\frac{25-29.9}{\text { Overweight }}$} & \multirow{2}{*}{$\frac{30-34.9}{\text { Grade } 1 \text { obesity }}$} & \multirow{2}{*}{$\begin{array}{l}\geq 35 \\
\text { Grade 2-3 obesity }\end{array}$} \\
\hline & & & & & & \\
\hline & & Mean \pm SD & Mean \pm SD & Mean \pm SD & Mean \pm SD & Mean \pm SD \\
\hline Fasting blood sugar (mg/dL) & $106.0 \pm 27.8$ & $96.7 \pm 19.7$ & $103.9 \pm 26.8$ & $109.0 \pm 28.4$ & $115.4 \pm 32.8$ & $122.3 \pm 39.9$ \\
\hline Systolic blood pressure (mmHg) & $135.3 \pm 19.7$ & $129.2 \pm 20.9$ & $133.8 \pm 19.7$ & $137.7 \pm 19.0$ & $141.0 \pm 19.3$ & $143.9 \pm 22.4$ \\
\hline Diastolic blood pressure $(\mathrm{mmHg})$ & $76.2 \pm 11.7$ & $72.3 \pm 12.1$ & $75.2 \pm 11.5$ & $77.8 \pm 11.6$ & $79.2 \pm 11.9$ & $81.1 \pm 14.0$ \\
\hline Triglyceride (mg/dL) & $126.8 \pm 85.0$ & $81.9 \pm 44.5$ & $118.6 \pm 82.9$ & $141.8 \pm 87.3$ & $152.3 \pm 89.7$ & $155.9 \pm 78.3$ \\
\hline Total cholesterol (mg/dL) & $199 \pm 36.7$ & $194.8 \pm 35.5$ & $199.4 \pm 36.3$ & $198.8 \pm 37.1$ & $198.9 \pm 37.6$ & $202.0 \pm 39.8$ \\
\hline GOT (U/L) & $25.1 \pm 14.7$ & $26.6 \pm 16.6$ & $24.6 \pm 14.2$ & $25.6 \pm 14.9$ & $27.4 \pm 16.9$ & $29.5 \pm 22.3$ \\
\hline GPT (U/L) & $23.4 \pm 19.7$ & $20.0 \pm 17.1$ & $21.8 \pm 18.7$ & $25.6 \pm 20.5$ & $28.9 \pm 23.6$ & $30.3 \pm 25.8$ \\
\hline Albumin (g/dL) & $4.3 \pm 0.3$ & $4.3 \pm 0.3$ & $4.3 \pm 0.3$ & $4.4 \pm 0.3$ & $4.3 \pm 0.3$ & $4.3 \pm 0.3$ \\
\hline BUN (mg/dL) & $17.6 \pm 6.4$ & $17.3 \pm 6.2$ & $17.4 \pm 6.4$ & $17.8 \pm 6.3$ & $18.4 \pm 8.1$ & $18.5 \pm 6.5$ \\
\hline Creatinine (mg/dL) & $1.0 \pm 0.5$ & $1.0 \pm 0.5$ & $1.0 \pm 0.5$ & $1.0 \pm 0.5$ & $1.0 \pm 0.6$ & $1.0 \pm 0.5$ \\
\hline Hemoglobin (g/dL) & $13.6 \pm 1.4$ & $12.9 \pm 1.5$ & $13.5 \pm 1.4$ & $13.8 \pm 1.4$ & $13.7 \pm 1.4$ & $13.6 \pm 1.4$ \\
\hline
\end{tabular}

BMI: body mass index; GOT: glutamic oxaloacetic transaminase; GPT: glutamic pyruvic transaminase; BUN: blood urine nitrogen.

doi:10.1371/journal.pone.0102589.t002

\section{Results}

The percentages of individuals in each BMI category, selected characteristics of all individuals, and mortality during follow-up are shown in Table 1 . The mean $\pm \mathrm{SD}$ age of participants was $73.1 \pm 6.6$ years. The 5 -year study period yielded 254,211 personyears of follow-up, with a mean follow-up of $3.3 \pm 1.3$ years. According to the WHO definition, the BMI of $3.5 \%, 57.8 \%$, $33.4 \%, 4.8 \%$, and $0.5 \%$ of the elderly population was classified as underweight, normal weight, overweight, grade 1 obesity, and grade 2-3 obesity, respectively. During the 5 years of follow-up, $3,842(5.0 \%)$ deaths occurred, including 877 (22.8\% of all deaths) deaths due to CVD and 1,116 (29.0\%) deaths due to expanded CVD. Among all participants, 9,923 (12.8\%), 30,745 (39.6\%), 9,349 (12.1\%), and 36,919 (47.6\%) had high FBS, SBP, TG, and TC, respectively; $57,166(73.7 \%)$ had at least one of these factors.

The clinical characteristics of each BMI category are presented in Table 2. The composition factors related to metabolic syndrome, including FBS, SBP, diastolic BP, and TG, were significantly higher with higher $\mathrm{BMI}$ (for FBS, $P<0.001$; for SBP, $P<0.001$; for diastolic BP, $P<0.001$; and for TG, $P<0.001$ ). Other laboratory data including glutamic oxaloacetic transaminase (GOT), glutamic pyruvic transaminase (GPT), albumin, blood urine nitrogen (BUN), creatinine, and hemoglobin also showed an association with $\mathrm{BMI}$ as the factors related to metabolic syndrome (for GOT, $P<0.001$; for GPT, $P<0.001$; for albumin, $P<0.001$; for BUN, $P<0.001$; for creatinine, $P<0.001$; and for hemoglobin, $P<0.001$ ).

The HRs of all-cause, CVD, and expanded CVD mortality calculated separately in the first (i.e., adequately adjusted) and second (i.e., over-adjusted) models are shown in Table 3. The predictors of mortality were generally similar between models. Underweight (all-cause mortality, HR: 1.92, 95\% confidence interval [CI]: 1.71-2.15; CVD mortality, HR: $1.74,95 \%$ CI: 1.36-2.23; expanded CVD mortality, HR: 1.77, 95\% CI: $1.42-$ 2.21) and grade $2-3$ obesity (all-cause mortality, HR: $1.59,95 \%$ CI: 1.06-2.38; CVD mortality, HR: 2.36, 95\% CI: 1.17-4.76; expanded CVD mortality, HR: 2.22, 95\% CI: 1.18-4.15) as well as older age, male sex, smoking, and high FBS were significant predictors of all-cause, GVD, and expanded CVD mortality. On the other hand, being married/cohabitating, higher education, alcohol consumption, more regular exercise, and high TC were inversely associated with mortality. The results of the sensitivity test with the Weibull model remain robust. The hazard ratios of non-GVD mortality (i.e., excluding those with CVD mortality) among each BMI category were also calculated, that is, underweight (HR: 1.97, 95\% CI: 1.73-2.24), overweight (HR: 0.81, 95\% CI: $0.74-0.88$ ), grade 1 obesity (HR: $0.84,95 \%$ CI: $0.69-1.03)$, and grade $2-3$ obesity (HR: 1.36 , 95\% CI: $0.83-2.24)$.

A sensitivity test combining grades 1 and 2-3 obesity as a single obese group was performed. Compared to normal body weight, this obese group (i.e., $\mathrm{BMI} \geq 30$ ) tended to have lower $\mathrm{HRs}$ for overall mortality (all-cause mortality, HR: 0.89, 95\% CI: $0.75-$ 1.04; GVD mortality, HR: 0.88, 95\% CI: 0.63-1.23; expanded CVD mortality, HR: 0.89, 95\% CI: 0.66-01.20). Another sensitivity test set the reference group as $23 \leq \mathrm{BMI}<25$, and the results were also similar.

The associations of BMI with all-cause, CVD, and expanded CVD mortality among the elderly were all represented as generally similar asymmetric U-shaped curves with large flat bottoms and right curves that started to rise significantly at $\mathrm{BMI} \geq$ 35 (Figure 1). The HRs of overweight and grade 1 obesity shared a nadir on the all-cause mortality curve, and the HR of grade 1 obesity was at the nadirs of the CVD and expanded CVD curves. The effects of grade 2-3 obesity were more prominent on CVD and expanded CVD mortality than all-cause mortality.

Subgroup analyses are presented in Figure 2. The HRs were almost the same in subgroup analyses. Among smokers, grade 2-3 obesity was more strongly associated with the mortality (all-cause mortality, HR: 3.25, 95\% CI: 1.33-7.93; GVD mortality, HR: 10.71, 95\% CI: 3.23-35.44; expanded GVD mortality, HR: 7.86, 95\% CI: 2.41-25.58). In the high TG group, underweight was strongly associated with mortality (all-cause mortality, HR: 5.82, 95\% CI: 3.30-10.25; CVD mortality, HR: 10.99, 95\% CI: $4.28-$ 28.22; expanded CVD mortality, HR: 14.22, 95\% CI: $7.18-$ 28.17). Among those with 3-4 factors related to metabolic syndrome, underweight was significantly associated with mortality 
Table 3. Cox Proportional Model of Factors Associated with All-cause, CVD, and Expanded CVD Mortality.

\begin{tabular}{|c|c|c|c|c|c|c|}
\hline \multirow{2}{*}{ Variables } & \multicolumn{2}{|c|}{ All-cause mortality } & \multicolumn{2}{|l|}{ CVD mortality } & \multicolumn{2}{|c|}{ Expanded CVD mortality } \\
\hline & $\mathrm{HR}^{\mathrm{a}}(95 \% \mathrm{Cl})$ & $\mathrm{HR}^{\mathrm{b}}(95 \% \mathrm{Cl})$ & $\mathrm{HR}^{\mathrm{a}}(95 \% \mathrm{Cl})$ & $\mathrm{HR}^{\mathrm{b}}(95 \% \mathrm{Cl})$ & $\mathrm{HR}^{\mathrm{a}}(95 \% \mathrm{Cl})$ & $\mathrm{HR}^{\mathrm{b}}(95 \% \mathrm{Cl})$ \\
\hline BMI (18.5-24.9) & 1.00 & 1.00 & 1.00 & 1.00 & 1.00 & 1.00 \\
\hline 15.0-18.4 (underweight) & $1.92(1.71-2.15)$ & $1.95(1.74-2.19)$ & $1.74(1.36-2.23)$ & $1.79(1.39-2.29)$ & $1.77(1.42-2.21)$ & $1.89(1.52-2.36)$ \\
\hline 25-29.9 (overweight) & $0.82(0.76-0.88)$ & $0.79(0.74-0.85)$ & $0.85(0.73-0.99)$ & $0.83(0.71-0.97)$ & $0.90(0.79-1.03)$ & $0.85(0.74-0.97)$ \\
\hline 30-34.9 (grade 1obesity) & $0.82(0.69-0.98)$ & $0.78(0.65-0.93)$ & $0.75(0.51-1.09)$ & $0.71(0.49-1.05)$ & $0.77(0.55-1.07)$ & $0.69(0.49-0.96)$ \\
\hline$\geq 35$ (grade $2-3$ obesity) & $1.59(1.06-2.38)$ & $1.48(0.99-2.21)$ & $2.36(1.17-4.76)$ & $2.24(1.11-4.52)$ & $2.22(1.18-4.15)$ & $1.94(1.03-3.63)$ \\
\hline Age (years) (65-69) & 1.00 & 1.00 & 1.00 & 1.00 & 1.00 & 1.00 \\
\hline $70-74$ & $1.58(1.39-1.78)$ & $1.55(1.37-1.76)$ & $1.71(1.30-2.25)$ & $1.69(1.28-2.22)$ & $1.82(1.43-2.31)$ & $1.78(1.40-2.26)$ \\
\hline $75-79$ & $2.65(2.37-2.97)$ & $2.58(2.30-2.89)$ & $3.34(2.62-4.31)$ & $3.27(2.55-4.20)$ & $3.34(2.69-4.16)$ & $3.23(2.59-4.03)$ \\
\hline $80-84$ & $4.33(3.86-4.86)$ & $4.19(3.73-4.70)$ & $5.22(4.05-6.72)$ & $5.07(3.93-6.53)$ & $5.23(4.19-6.54)$ & $5.06(4.05-6.33)$ \\
\hline$\geq 85$ & $7.60(6.74-8.58)$ & $7.33(6.49-8.28)$ & $10.26(7.90-13.33)$ & $9.93(7.64-12.92)$ & $9.18(7.26-11.59)$ & $8.88(7.02-11.24)$ \\
\hline Sex (Female) & 1.00 & 1.00 & 1.00 & 1.00 & 1.00 & 1.00 \\
\hline Male & $2.08(1.92-2.24)$ & $1.97(1.82-2.13)$ & $1.71(1.46-2.01)$ & $1.68(1.43-1.98)$ & $1.68(1.46-1.94)$ & $1.64(1.42-1.89)$ \\
\hline Marital status (single) & 1.00 & 1.00 & 1.00 & 1.00 & 1.00 & 1.00 \\
\hline Married/cohabiting & $0.83(0.77-0.89)$ & $0.83(0.78-0.89)$ & $0.82(0.70-0.94)$ & $0.82(0.71-0.94)$ & $0.79(0.69-0.89)$ & $0.79(0.69-0.90)$ \\
\hline Education (years) (None) & 1.00 & 1.00 & 1.00 & 1.00 & 1.00 & 1.00 \\
\hline $1-6$ & $0.84(0.75-0.93)$ & $0.85(0.76-0.94)$ & $0.87(0.70-1.09)$ & $0.88(0.70-1.10)$ & $0.89(0.73-1.08)$ & $0.90(0.74-1.09)$ \\
\hline $6-12$ & $0.71(0.64-0.79)$ & $0.72(0.65-0.80)$ & $0.73(0.59-0.92)$ & $0.74(0.59-0.93)$ & $0.69(0.57-0.84)$ & $0.71(0.58-0.86)$ \\
\hline$\geq 12$ & $0.56(0.50-0.63)$ & $0.57(0.51-0.64)$ & $0.63(0.49-0.80)$ & $0.64(0.50-0.81)$ & $0.60(0.48-0.74)$ & $0.61(0.50-0.76)$ \\
\hline Smoking (None) & 1.00 & 1.00 & 1.00 & 1.00 & 1.00 & 1.00 \\
\hline Frequently/occasionally & $1.48(1.34-1.63)$ & $1.48(1.35-1.63)$ & $1.30(1.04-1.61)$ & $1.30(1.05-1.62)$ & $1.29(1.06-1.57)$ & $1.30(1.07-1.57)$ \\
\hline Alcohol consumption (None) & 1.00 & 1.00 & 1.00 & 1.00 & 1.00 & 1.00 \\
\hline Frequently/occasionally & $0.72(0.65-0.78)$ & $0.73(0.66-0.80)$ & $0.66(0.54-0.81)$ & $0.67(0.55-0.82)$ & $0.63(0.53-0.76)$ & $0.64(0.54-0.77)$ \\
\hline Regular exercise (None) & 1.00 & 1.00 & 1.00 & 1.00 & 1.00 & 1.00 \\
\hline 1-2 times/week & $0.68(0.62-0.75)$ & $0.69(0.63-0.75)$ & $0.72(0.59-0.87)$ & $0.72(0.59-0.87)$ & $0.71(0.60-0.83)$ & $0.71(0.60-0.84)$ \\
\hline 3-5 times/week & $0.53(0.49-0.58)$ & $0.53(0.49-0.58)$ & $0.59(0.49-0.72)$ & $0.59(0.49-0.72)$ & $0.53(0.45-0.63)$ & $0.54(0.46-0.64)$ \\
\hline High FBS (None) & & 1.00 & & 1.00 & & 1.00 \\
\hline$\geq 126 \mathrm{mg} / \mathrm{dL}$ & & $1.44(1.32-1.57)$ & & $1.23(1.02-1.48)$ & & $1.80(1.55-2.09)$ \\
\hline High SBP (None) & 1.00 & & 1.00 & & 1.00 & \\
\hline$\geq 140 \mathrm{mmHg}$ & & $1.04(0.98-1.11)$ & & $1.13(0.99-1.29)$ & & $1.11(0.99-1.25)$ \\
\hline High TG (None) & & 1.00 & & 1.00 & & 1.00 \\
\hline$\geq 200 \mathrm{mg} / \mathrm{dL}$ & & $1.02(0.92-1.13)$ & & $1.05(0.85-1.30)$ & & $1.18(0.98-1.40)$ \\
\hline High TC (None) & & 1.00 & & 1.00 & & 1.00 \\
\hline$\geq 200 \mathrm{mg} / \mathrm{dL}$ & & $0.77(0.72-0.83)$ & & $0.89(0.77-1.02)$ & & $0.84(0.75-0.96)$ \\
\hline
\end{tabular}

aModel 1: adjusted for age, sex, marital status, education level, smoking, alcohol consumption, and exercise status.

${ }^{\mathrm{b}}$ Model 2: adjusted for all factors in Model 1 plus high FBS, high SBP, high TG, and high TC.

BMI: body mass index; CVD: cardiovascular disease; FBS: fasting blood sugar; SBP: systolic blood pressure; TG: triglycerides; TC: total cholesterol.

doi:10.1371/journal.pone.0102589.t003

(all-cause mortality, HR: 4.86, 95\% CI: 2.36-10.01; CVD mortality, HR: 12.72, 95\% CI: 4.31-37.51; expanded CVD mortality, HR: 11.42, 95\% CI: 4.73-27.58).

\section{Discussion}

The results of this study show the association between measured BMI and mortality in the elderly. The large sample size enabled subgroup analysis according to particular characteristics. Relative to the normal weight category, the associations of BMI with allcause, GVD, and expanded GVD mortality in the elderly were generally represented as U-shaped curves with large flat bottoms at overweight and grade 1 obesity, and a right curve that was significantly elevated at a $\mathrm{BMI} \geq 35$.
The different effects of grades 1 and 2-3 obesity on mortality are noteworthy. When subjects with $\mathrm{BMI} \geq 30$ were treated as a single obesity group, the overall HR indicated lower mortality; this is because mildly obese subjects outnumbered moderate-severe obese subjects. These results are broadly consistent with those of 3 meta-analyses, which report obesity is associated with lower mortality $[13,14,18]$. However, treating different levels of obesity as a single group might obscure the heterogeneous effects of mild and moderate-severe obesity on mortality.

Some authors suggest lowering the BMI cut-offs for Asian populations [19], while others disagree [20]. Wen et al. report significant mortality risks starting at a $\mathrm{BMI} \geq 25$ in a much younger Taiwanese cohort [19]. Meanwhile, $\mathrm{Gu}$ et al. report underweight and obesity are associated with increased mortality in a Chinese 


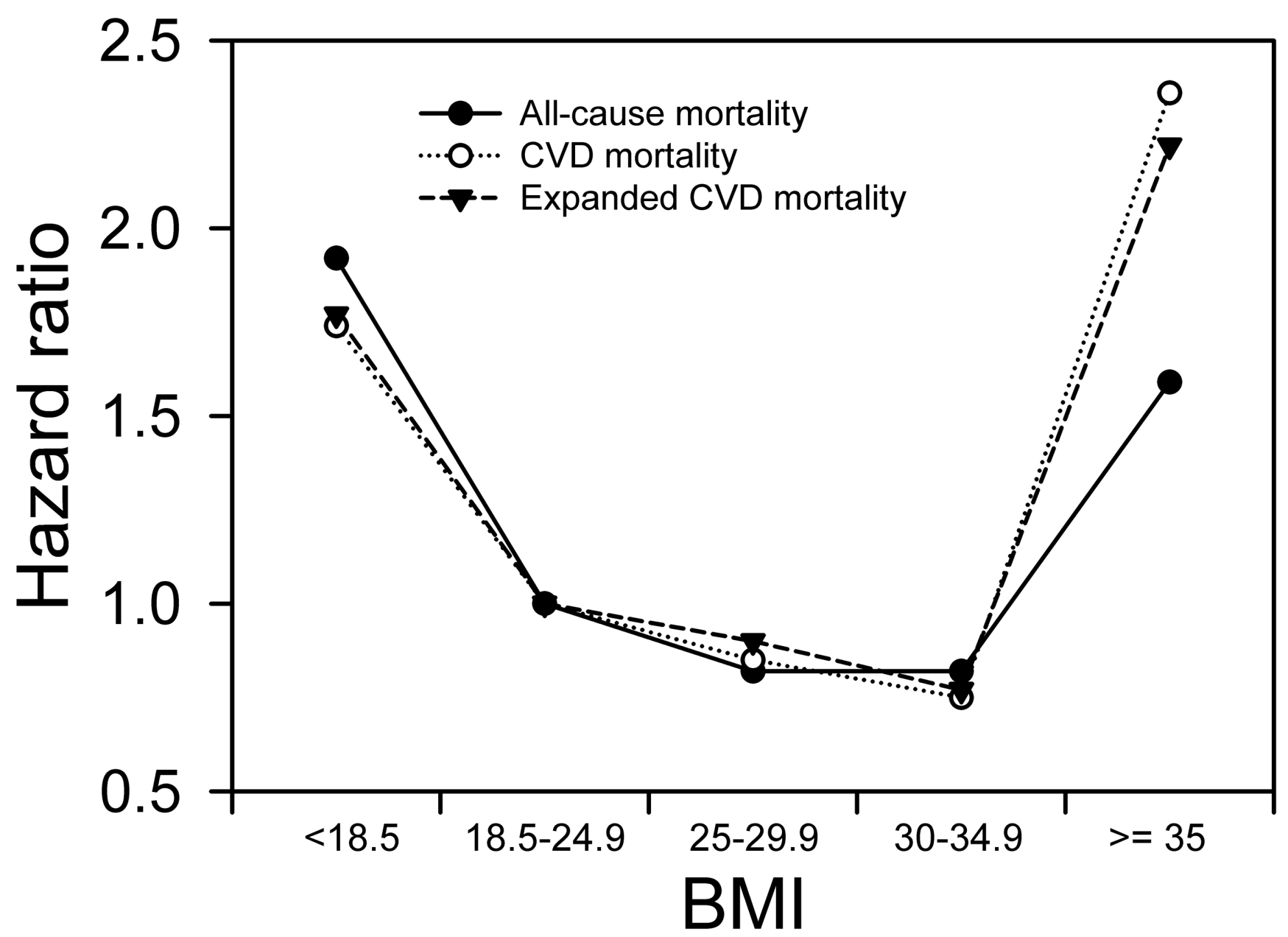

Figure 1. HRs for all-cause, CVD, and expanded CVD mortality according to BMI category. doi:10.1371/journal.pone.0102589.g001

adult population and support the use of a single common recommendation for defining overweight and obesity for all ethnicities [20]. In the present study, the risks of mortality in an elderly Taiwanese population did not differ substantially from those of Western counterparts [13,14], corroborating the use of a single common recommendation for defining overweight and obesity among all ethnic groups. Furthermore, these findings corroborate the notion that overweight and mild obesity are relatively less harmful in the elderly.

Underweight was associated with increased mortality in previous studies [21-23]. In the present study, underweight was associated with all-cause, CVD, and expanded CVD mortality in the elderly. These effects persisted in subgroup analysis. Accordingly, some studies suggest modestly higher body weight may improve survival in some circumstances [5-10]. Indeed, overweight and grade 1 obesity were associated with lower mortality risks in the present study, suggesting slightly or mildly elevated BMI increases survival in the elderly. There are some possible explanations for this phenomenon. First, fat storage might be a protective resource in some individuals exposed to acute insults or chronic wasting [7]. Second, BMI cannot distinguish excess body fat from increased lean mass [24]. Third, obesity patients may present with symptoms and morbidities earlier [25], making them more likely to receive prompt medical treatment [26].
Nevertheless, the observed decrease in mortality risk reverses beyond mild obesity. In particular, grade 2-3 obesity was associated with increased risks of all-cause, CVD, and expanded CVD mortality. These results are consistent with those of a previous study, reporting moderate obesity is associated with an increased risk of mortality [13]. Therefore, the present findings may have important implications contrary to the current clinical recommendation that overweight and mildly obese elderly people should lose weight.

In previous studies investigating the association between BMI and mortality, models were considered adequate if adjusted for age, sex, and smoking. Models adjusting for additional factors such as hypertension are considered possibly over-adjusted, because such factors are considered to be a part of the causal relationship between obesity and mortality [14]. Therefore, in order to test the predictive capability of BMI for mortality rather than inferring a causal relationship, evaluating other factors as potential confounders is not a large concern. Regardless, in the present study, the results of the adequately adjusted and over-adjusted models were not substantially different.

Smoking is considered an important risk factor for morbidity and mortality, particularly CVD mortality. Previous studies have analyzed the association between BMI and mortality among nonsmokers to avoid the confounding effect of smoking [27]. In the present subgroup analysis, the results of non-smokers were similar 


\section{All-cause mortality}
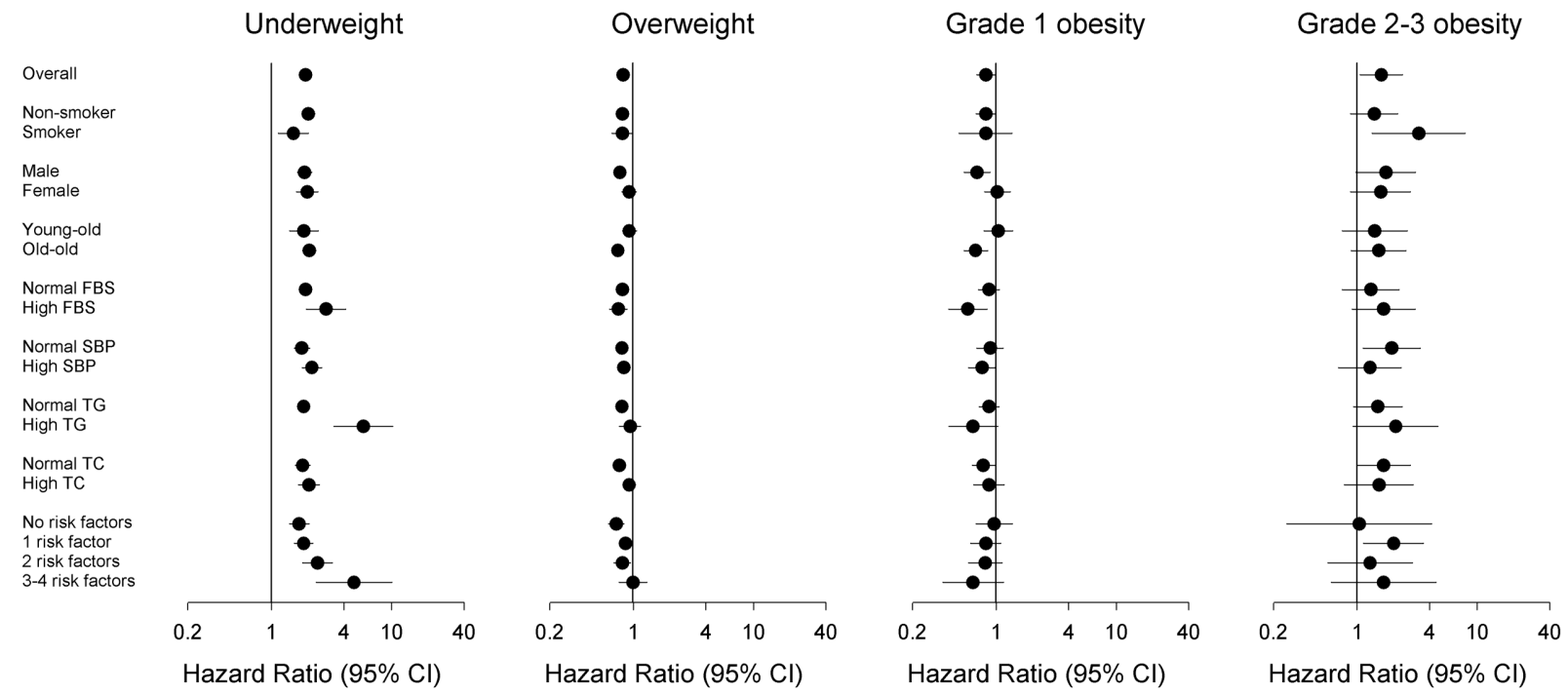

CVD mortality
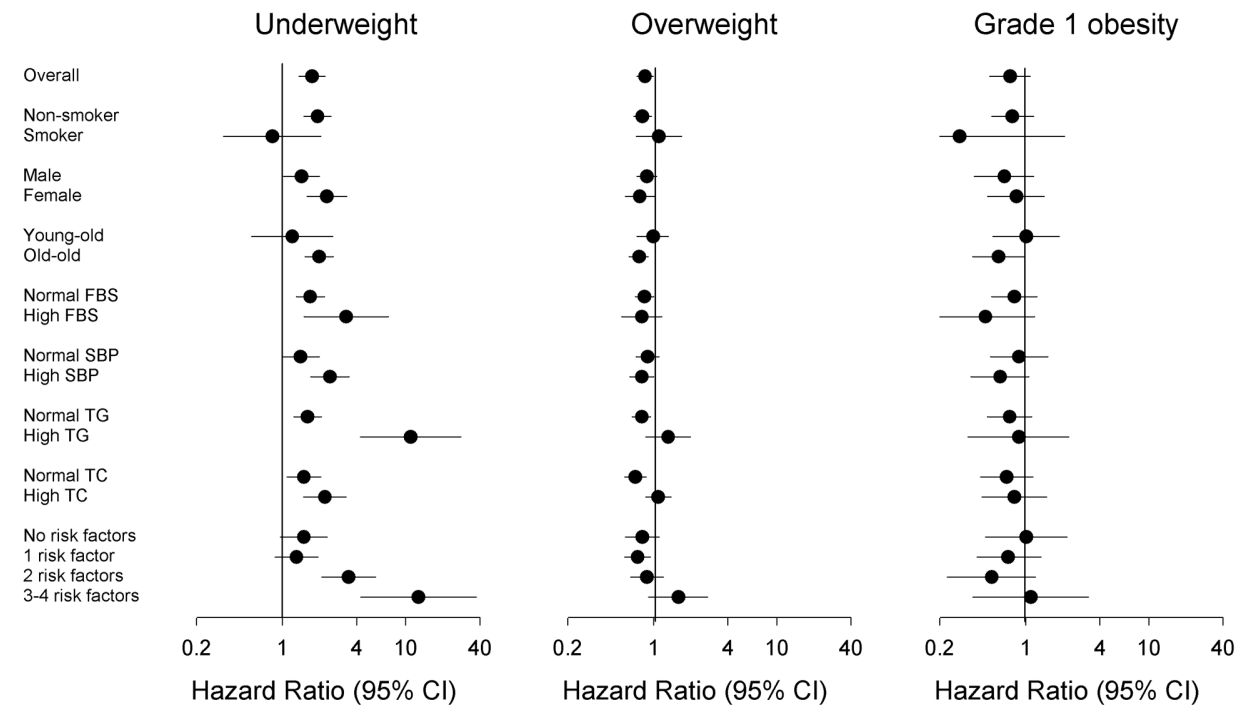

Grade 2-3 obesity

Expanded CVD mortality

Overweight

Grade 1 obesity

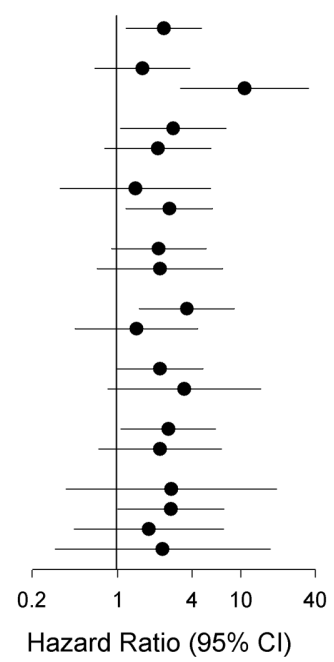

\section{Underweight}

Overall

Non-smoker
Smoker

Male
Female

Young-old

Old-old

Normal FBS

High FBS

Normal SBP

Normal TG

High TG

Normal TC

High TC

No risk factors

1 risk factor

3-4 risk factors
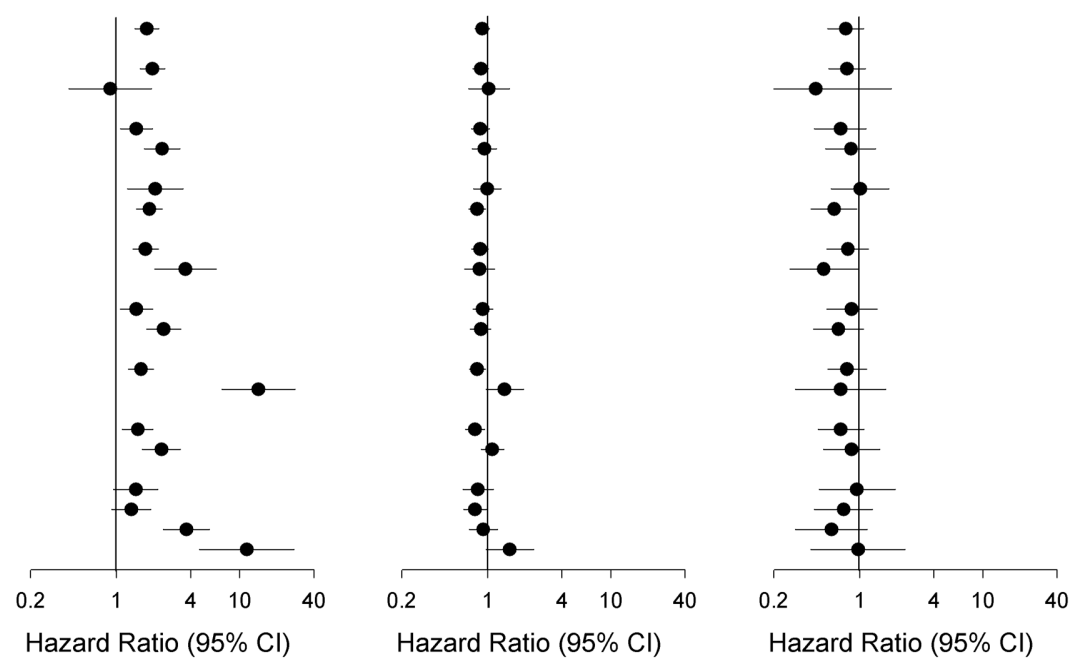

Grade 2-3 obesity

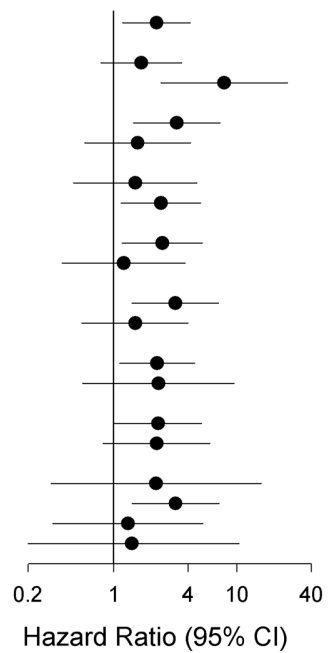


Figure 2. Subgroup analysis of all-cause, CVD, and expanded CVD mortality. doi:10.1371/journal.pone.0102589.g002

to those of the total population. However, it is worth noting the HRs of smokers increased sharply in grade 2-3 obesity, for especially CVD and expanded CVD mortality. The excess mortality risks of BMI among smokers suggest smoking enhances the relationship between higher BMI and survival in the elderly [28].

Diabetes is one of the most significant risk factors for CVD, and BMI is strongly associated with an increased prevalence of CVD independent of metabolic syndrome [29]. In the present study, high FBS was significantly associated with all-cause, CVD, and expanded CVD mortality, whereas high SBP and TG were not. Most interestingly, high TC was negatively associated with mortality, which has been reported previously [30,31]. In the present study, high TC was associated with higher BMI. Therefore, high TC is possibly associated with better global health conditions among the elderly population. However, the actual role of hypercholesterolemia as a risk factor for mortality among the elderly requires further investigation.

The present study has several limitations. First, this study did not consider preexisting diseases or therapeutic regimens used to control diabetes, hypertension, or dyslipidemia. Baseline laboratory data were used for adjustment in the models and subgroup analysis. Moreover, reverse causation and illness-related weight loss could confound the association between BMI and mortality [32]. A sensitivity test to set the reference group as subjects with a BMI from 23-24.9, which would exclude people who might be underweight due to underlying diseases, would partially reduce the impact of preexisting diseases as the cause of death. Regardless, residual confounding or reverse causation due to preexisting illnesses is reported to have little effect on the estimated relative risks of mortality [8,33]. Second, the baseline examination data

\section{References}

1. Flegal KM, Carroll MD, Ogden CL, Johnson CL (2002) Prevalence and trends in obesity among US adults, 1999-2000. JAMA 288: 1723-1727.

2. Kumanyika SK, Obarzanek E, Stettler N, Bell R, Field AE, et al. (2008) Population-based prevention of obesity: the need for comprehensive promotion of healthful eating, physical activity, and energy balance: a scientific statement from American Heart Association Council on Epidemiology and Prevention, Interdisciplinary Committee for Prevention (formerly the expert panel on population and prevention science). Circulation 118: 428-464.

3. Lavie CJ, Milani RV, Ventura HO (2008) Untangling the heavy cardiovascular burden of obesity. Nat Clin Pract Cardiovasc Med 5: 428-429.

4. Yazdanyar A, Newman AB (2009) The burden of cardiovascular disease in the elderly: morbidity, mortality, and costs. Clin Geriatr Med 25: 563-577, vii.

5. Artham SM, Lavie CJ, Milani RV, Ventura HO (2008) The obesity paradox: impact of obesity on the prevalence and prognosis of cardiovascular diseases. Postgrad Med 120: 34-41.

6. Auyeung TW, Lee JS, Leung J, Kwok T, Leung PC, et al. (2010) Survival in older men may benefit from being slightly overweight and centrally obese-a 5year follow-up study in 4,000 older adults using DXA. J Gerontol A Biol Sci Med Sci 65: 99-104.

7. Doehner W, Clark A, Anker SD (2010) The obesity paradox: weighing the benefit. Eur Heart J 31: 146-148.

8. Greenberg JA (2013) The obesity paradox in the US population. Am J Clin Nutr 97: 1195-1200.

9. Hastie CE, Padmanabhan S, Slack R, Pell AC, Oldroyd KG, et al. (2010) Obesity paradox in a cohort of 4880 consecutive patients undergoing percutaneous coronary intervention. Eur Heart J 31: 222-226.

10. Uretsky S, Messerli FH, Bangalore S, Champion A, Cooper-Dehoff RM, et al. (2007) Obesity paradox in patients with hypertension and coronary artery disease. Am J Med 120: 863-870.

11. Stevens J, CaiJ, Pamuk ER, Williamson DF, Thun MJ, et al. (1998) The effect of age on the association between body-mass index and mortality. N Engl J Med 338: $1-7$.

12. Masters RK, Powers DA, Link BG (2013) Obesity and US mortality risk over the adult life course. Am J Epidemiol 177: 431-442.

13. Janssen I, Mark AE (2007) Elevated body mass index and mortality risk in the elderly. Obes Rev 8: 41-59. were used to represent all participants in the cohort. These data may have changed over time, leading to complex effects on mortality rates. However, the present results demonstrate the power of a single determination of BMI for predicting mortality risk. Third, the present study analyzed the statistical association between BMI and mortality. Several other factors associated with weight and mortality, such as physical activity level and body composition, could be responsible for part of the observed association. Therefore, mortality data from well-controlled weight loss trials are required to clarify the association between BMI and mortality. Fourth, the voluntary participants of this study may not be representative of the general population. However, because risk comparison was based on internal comparison, the calculated relative risks are a reasonable estimate of those in the general population.

In conclusion, the U-shaped relationship between BMI and mortality in the elderly in the present study suggests unilateral promotions or interventions in weight reduction in the elderly may be inappropriate. Thus, losing weight may not be uniformly beneficial to the health of elderly persons. In particular, it might be necessary to reevaluate strategies related to the pertinence of lowering the weight of those overweight and grade 1 obesity elderly patients.

\section{Author Contributions}

Conceived and designed the experiments: CYW YCG NH YJC HYH CPL. Performed the experiments: CYW YCG NH HYH. Analyzed the data: CYW YCG NH YJC HYH CPL. Contributed reagents/materials/ analysis tools: CYW NH HYH YJC CPL. Wrote the paper: CYW CPL.

14. Flegal KM, Kit BK, Orpana H, Graubard BI (2013) Association of all-cause mortality with overweight and obesity using standard body mass index categories: a systematic review and meta-analysis. JAMA 309: 71-82.

15. Lin WY, Albu J, Liu CS, Huang HY, Pi-Sunyer FX, et al. (2010) Larger body mass index and waist circumference are associated with lower mortality in Chinese long-term care facility residents. J Am Geriatr Soc 58: 2092-2098.

16. Flegal KM, Graubard BI, Williamson DF, Gail MH (2010) Sources of differences in estimates of obesity-associated deaths from first National Health and Nutrition Examination Survey (NHANES I) hazard ratios. Am J Clin Nutr 91: 519-527.

17. Department of Health, Taipei City Government. Available: http://www.health. gov.tw/Default.aspx?tabid = 401. Accessed 2014 April 1 .

18. McGee DL (2005) Body mass index and mortality: a meta-analysis based on person-level data from twenty-six observational studies. Ann Epidemiol 15: 8797.

19. Wen CP, David Cheng TY, Tsai SP, Chan HT, Hsu HL, et al. (2009) Are Asians at greater mortality risks for being overweight than Caucasians? Redefining obesity for Asians. Public Health Nutr 12: 497-506.

20. Gu D, He J, Duan X, Reynolds K, Wu X, et al. (2006) Body weight and mortality among men and women in China. JAMA 295: 776-783.

21. Flegal KM, Graubard BI, Williamson DF, Gail MH (2007) Cause-specific excess deaths associated with underweight, overweight, and obesity. JAMA 298: 20282037.

22. Nagai M, Kuriyama S, Kakizaki M, Ohmori-Matsuda K, Sugawara Y, et al. (2010) Effect of age on the association between body mass index and all-cause mortality: the Ohsaki cohort study. J Epidemiol 20: 398-407.

23. Wang Y, Qain Y, Zhang J, Tang X, Sun J, et al. (2013) Association of body mass index with cause specific deaths in chinese elderly hypertensive patients: minhang community study. PLoS One 8: e71223.

24. Heymsfield SB, Cefalu WT (2013) Does body mass index adequately convey a patient's mortality risk? JAMA 309: 87-88.

25. Oreopoulos A, McAlister FA, Kalantar-Zadeh K, Padwal R, Ezekowitz JA, et al. (2009) The relationship between body mass index, treatment, and mortality in patients with established coronary artery disease: a report from APPROACH. Eur Heart J 30: 2584-2592. 
26. Schenkeveld L, Magro M, Oemrawsingh RM, Lenzen M, de Jaegere P, et al. (2012) The influence of optimal medical treatment on the 'obesity paradox', body mass index and long-term mortality in patients treated with percutaneous coronary intervention: a prospective cohort study. BMJ Open 2: e000535.

27. Tsai SP, Wen CP, Chan HT, Chiang PH, Tsai MK, et al. (2008) The effects of pre-disease risk factors within metabolic syndrome on all-cause and cardiovascular disease mortality. Diabetes Res Clin Pract 82: 148-156.

28. Whitlock G, Lewington S, Sherliker P, Clarke R, Emberson J, et al. (2009) Bodymass index and cause-specific mortality in 900000 adults: collaborative analyses of 57 prospective studies. Lancet 373: 1083-1096.

29. He Y, Jiang B, Wang J, Feng K, Chang Q et al. (2007) BMI versus the metabolic syndrome in relation to cardiovascular risk in elderly Chinese individuals. Diabetes Care 30: 2128-2134.
30. Akerblom JL, Costa R, Luchsinger JA, Manly JJ, Tang MX, et al. (2008) Relation of plasma lipids to all-cause mortality in Caucasian, African-American and Hispanic elders. Age Ageing 37: 207-213.

31. Schupf N, Costa R, Luchsinger J, Tang MX, Lee JH, et al. (2005) Relationship between plasma lipids and all-cause mortality in nondemented elderly. $\mathrm{J}$ Am Geriatr Soc 53: 219-226.

32. Flegal KM, Graubard BI, Williamson DF, Cooper RS (2011) Reverse causation and illness-related weight loss in observational studies of body weight and mortality. Am J Epidemiol 173: 1-9.

33. Flegal KM, Graubard BI, Williamson DF, Gail MH (2007) Impact of smoking and preexisting illness on estimates of the fractions of deaths associated with underweight, overweight, and obesity in the US population. Am J Epidemiol 166: 975-982 